Flavio Pajer

Salesian Pontifical University - Rome, Italy

\title{
L'educare e il credere tra vecchie e nuove utopie
}

\author{
Education and Faith between OId and New Utopias
}

\section{Abstract}

The article focuses three problematic knots. The first one underlines how the modern utopias are not dead, but they have strikingly moved: the modern ideal of freedom, for example, changed into the post-modern ideal of security; the monism of values shared in common turned into a pluralism of personal, discontinuous and fragmented choices. The second knot identifies education itself as an evolving utopia: from the post colonial dream of education for all to the present priority to educate ex novo the social links in a globalised world; from the society of knowledge (instrumental good) to a pluralistic society of living together (structural good). The third knot calls to mind the classical eschatological hendiadys of the "already and not yet" that the theology of Christian Hope propose once more as the paradigm of the human living. In the - political and at same time mystic - dynamic of the historical present, dissuading in this way the believers from looking for the salvation in easy and utopian projections beyond this world.

\section{Keywords}

Education, faith, the modern and post-modern utopias.

\section{Le utopie oggi? Non sono morte, si sono spostate}

Dai tempi di Freud il pendolo dei sogni umani si è spostato dal desiderio di maggior libertà a quello di maggior sicurezza. Se ieri prevaleva la richiesta di uguaglianza, oggi emerge la voglia di parità, la garanzia di vedersi riconosciuto il diritto di scegliere il proprio modo di vivere. 
E persino l'istanza di solidarietà, tanto in auge nelle società opulente delle democrazie occidentali, si sta estenuando nell'ideale di 'rete', di comunità virtuale tanto accessibile quanto aleatoria. Sicurezza, Parità, Rete: questi, in sostanza, i sostituti odierni dei mitici valori di Libertà, Uguaglianza, Fraternità sbandierati dalle barricate del 1789 parigino. Chi lo afferma è Zygmunt Bauman, una delle 'antenne' culturali più attente alle tendenze contemporanee, e lo afferma in un'intervista rilasciata all'inizio del $2010^{1}$.

Sono morte dunque le utopie della modernità? No, risponde Bauman, si sono semplicemente spostate. e spiega come: "La vita moderna è segnata da un'ossessiva e compulsiva trasformazione di ogni cosa in qualcosa d'altro e di migliore della versione precedente della medesima cosa. Anche la presente è una vita che non è possibile senza utopia. Ecco perché gli annunci che danno per morta l'utopia sono prematuri. Però, qualcosa è cambiato. Dall'utopia è sparito il topos: la visione di un luogo. Anche l'utopia è stata privatizzata. Le utopie odierne non parlano più di un Paese gestito bene, ma semmai di un miglioramento della mia situazione individuale, qui e ora. La speranza di un avvenire migliore non la associamo più a un saggio legislatore $\mathrm{o}$ a uno sforzo solidale, ma alla nostra furbizia, alla nostra arte di arrangiarsi”. In altre parole, è finito l'ordine mondiale basato sulla formula: Stato Nazione Territorio. E’ finito il tempo in cui politici e sociologi definivano la società come "l'unità di misura più alta e più grande a cui la gente è iscritta" e nella quale i confini della società coincidevano con quelli dello Stato-nazione. Oggi le funzioni che una volta svolgeva lo Stato sono state assunte (scippate?) dal Mercato.

L'appartenenza alla società è presto e spesso sostituita dai fragili legami costruiti su facebook o con twitter: Una comunità che è facile creare velocemente ed altrettanto facile smantellare rapidamente. Anzi, c'è già chi, sazio di social network e geloso di un riconquistato senso della privacy, vuol sparire dal web per emanciparsi dalle spire del neo-feudalesimo virtuale di internet, dove a farla da padroni sono i pochi manager della civiltà digitale transnazionale e transmediale. Eloquente la metafora del guardaroba suggerita in proposito ancora da Bauman: "Tutto avviene come quando in un teatro gli spettatori lasciano in guardaroba i soprabiti, e poi finito lo show li ritirano, e ognuno va per la propria strada. Ecco, le comunità oggi sono delle 'comunità da guardaroba'. La comunità tradizionale che chiede e impone delle regole, che include e stabilisce le frontiere, oggi non è accettabile: troppe sono le seduzioni cui siamo esposti, convinti come siamo che scegliere sia il nostro diritto eterno".

${ }^{1}$ Z. Bauman, a colloquio con Wlodek Goldkorn, nello 'speciale’ Restart 2010: Riprendiamoci le nostre utopie, "L’Espresso” 7 gennaio 2010, p. 100-103. 
Il nostro Gianni Vattimo non era lontano da queste posizioni quando, qualche anno addietro, proprio all'indomani dell'11 settembre, in una sede prestigiosa come quella dell'UNESCO, si domandava, al seguito di altri filosofi del Novecento: ma noi occidentali, stiamo andando davvero verso un crepuscolo dei valori? ${ }^{2}$ La sua risposta partiva dal superamento dell'ormai sterile bipolarità tra valori eurocentrici e valori universalistici, tra etnocentrismo e cosmopolitismo, per appoggiare hegelianamente una terza via, quella del negoziato che rispetti le diverse tradizioni culturali e valoriali senza però tradire la nostra storia, la nostra biografia culturale. "Noi occidentali - scrive Vattimo - non possiamo imporre ai paesi terzi il nostro atteggiamento crepuscolare verso i valori. Questo atteggiamento può e deve realizzarsi anzitutto in noi, per farci evitare l'errore fatale di credere che il solo modo di far fronte al fondamentalismo degli altri sia quello di diventare fondamentalisti noi stessi". Tendenza che il filosofo individua talora, e condanna, nella posizione "delle Chiese e di certi intellettuali democratici di sinistra quando ci invitano - in nome di un realismo tragico alla Benjamin o alla Carl Schmitt - a ritrovare una passione 'autentica' per i valori. Vorrebbero imporci una terapia di ringiovanimento artificiale, quando questo non potrà portare dritto che alla violenza e alla guerra".

E' molto probabile che l'utopia oggi vada cercata proprio in questa nuova maniera di vivere i valori. In fondo le guerre del passato erano simmetricamente speculari dal punto di vista della psicologia collettiva: gli eserciti in campo condividevano più o meno la stessa fede negli stessi valori (talora la stessa fede religiosa!). E' vero: oggi, noi occidentali, consapevoli o meno, ai valori e agli ideali della nostra tradizione "non ci crediamo più", mentre i nostri 'dirimpettai' non sempre e non necessariamente fanatici o terroristi - sembrano pronti a morire per difendere i loro valori. Cedere però alla tentazione dello scontro frontale significherebbe ripiombare nella giungla, ridiventare violenti e soprattutto "propter vitam vivendi perdere causas", cioè ostinarsi a sopravvivere a costo di rinunciare a ciò che costituisce la nostra vita stessa, non già in senso biologico ma 'biografico' ed etico. In tal senso, dunque, il focus dell'utopia collettiva si sposta pragmaticamente dalla concezione aggressiva di un unico universo simbolico (monismo dei valori) a una concezione dialogale, interlocutoria, di un pluriverso di patrimoni simbolici (politeismo dei valori). Concezione, questa seconda, che esige una ridistribuzione dei "beni simbolici" tra le varie culture, perché nessuna si arroghi il diritto di averne la perenne ed esclusiva proprietà.

${ }^{2}$ G. Vattimo, Vers un crépuscule des valeurs?, in: J. Bindé (ed.), Où vont les valeurs? Entretiens du XXIè siècle, Editions UNESCO/Albin Michel, Paris 2004, pp. 29-36. 
E' qui che si innesta, con ogni evidenza, il problema capitale della trasmissione del patrimonio di beni simbolici, tra i quali quelli veicolati dalle tradizioni religiose, che ne costituiscono il tessuto identitario, ma che spesso sono serviti (e asserviti) a modellare l'ethos di intere società civili. Detto problema mi sembra oggi formulabile in questi termini: ci troviamo tutti - come individui, come società, come chiese - in una posizione di Giano bifronte: da una parte guardiamo verso il passato archeologico del nostro essere e del senso del nostro essere (verso l'arché, le radici, la tradizione, i topoi che hanno forgiato le nostre identità), dall'altra non possiamo non guardare al télos del nostro futuro, o meglio all'u-topos del nostro destino. Si impone una armonizzazione interattiva delle due "visioni", una reciproca e simmetrica ricostruzione del patrimonio, in modo da evitare sia la riduzione mortifera dei valori a quelli collaudati da un passato già 'consumato', sia la fuga velleitaria nelle utopie irrealizzabili di un futuro solo sognato.

L'educazione dell'uomo si gioca in questo tiro alla fune tra passato e futuro, tra un già e un non-ancora, come ripetono con illuminante intuizione non pochi teologi contemporanei del messianismo biblico e dell'escatologia cristiana. Si gioca, per dirla con lo stupore di Pascal, tra un tutto e un niente, tra una "capacità d'infinito" e una miseria altrettanto infinita, tra una nostalgia inappagata di perfezione e l'inesaorabile finitudine. Al punto che la stessa educazione dell'uomo si rivela immancabilmente una utopia: una generosa ipotesi anticipatrice destinata però a ridimensionarsi con la libera resistenza dell'educando. Chi infatti dà diritto all'uomo di manomettere la coscienza, la libertà e il destino di un altro uomo? Ogni figlio è utopa per i genitori. Ogni discepolo è utopia per l'insegnante. Ogni neofita è utopia per il maestro di spirito. Ma se il sogno dell'adulto non è condiviso dal minore, rimane sogno e alla fine si muta in delusione. In tempo di 'emergenza educativa' ridiventa prioritario educarsi ed educare alla speranza ${ }^{3}$.

\section{Anche l'utopia «educazione» si va spostando}

Chi non ricorda i profeti della "nuova educazione" dei gloriosi anni SessantaSettanta? Venne don Milani a ritentare sui colli di Barbiana, in polemica con la scuola selettiva, l'utopia della democratizzazione del sapere, della "scuola di tutti e per tutti”. Venne Ivan Illich a predicare, nel tempo dell'iperscolarizzazione, il sogno della descolarizzazione in una "società conviviale", nell'intento di sottrarre le persone asservite alla sudditanza dal monopolio economico e dal sistema

\footnotetext{
${ }^{3}$ Cf. A.M.Favorini (ed.), Educare alla speranza, FrancoAngeli, Milano 2010.
} 
politico. Venne Paulo Freire a proporre, nel continente delle favelas suburbane e dei campesinos andini, l'educazione come pratica di libertà, come processo comunitario di coscientizzazione e liberazione, dove gli oppressi emergenti dal rovescio della storia avrebbero dovuto riscattarsi come protagonisti autonomi della propria condizione storica ${ }^{4}$. La dichiarazione universale dei diritti umani aveva appena vent'anni o poco più, ma cominciava a dare i suoi frutti.

E sul finire del millennio, una volta spentesi una dopo l'altra le voci carismatiche dei profeti solitari e 'alternativi', ecco imporsi - ma questa volta con la forza pianificatrice delle istituzioni nazionali e internazionali - l'annuncio di un'altra utopia educativa, quella dell'educazione permanente e globale ${ }^{5}$, dell'educazione ai valori della democrazia e della convivenza, dell'educazione alla diversità culturale e alla giustizia sociale, dell'educazione al futuro... Se ne fece portabandiera il presidente della Commissione europea, Jacques Delors, che dopo aver consultato per tre anni un gruppo di massimi specialisti dei cinque continenti, consegna in un "libro bianco" dell'Unesco le linee programmatiche "sull'educazione per il XXI secolo" 6 . Un disegno strategico grandioso e generoso quello lanciato ai responsabili delle politiche educative e culturali del pianeta: l'istruzione e l'educazione - vi si afferma - sono oggi l'utopia necessaria per imparare a vivere insieme nel villaggio globale, l'utopia per creare un mondo dallo sviluppo sostenibile, dove i popoli vivano in democrazia e solidarietà, e dove siano superate alcune forti tensioni come quelle esistenti tra il locale e il globale, l'individuale e l'universale, la tradizione e la modernità, il bisogno di competizione e l'esigenza di solidarietà, l'espansione delle conoscenze e la capacità di assimilarle, i valori spirituali-morali e quelli materiali.. Siffatta educazione del cittadino, concludeva Delors, per essere idonea all'immane compito, dovrà basarsi su quattro pilastri: "imparare a conoscere, imparare ad agire, imparare a vivere insieme, imparare ad essere”.

Il terzo di questi quattro pilastri - "imparare a vivere insieme" - suona nuovo e insolito. Non era comune toccare questo tasto nelle legislazioni scolastiche o nella letteratura pedagogica degli ultimi decenni. Segno che il "vivere insieme" non è più scontato nelle società d'oggi. Dopo la stagione delle società-nazione,

\footnotetext{
${ }^{4}$ Come riferimenti emblematici di quella stagione mi limito a segnalare questi tre "classici" della pedagogia alternativa: L.Milani, Lettera a una professoressa, 1967; I.Illich, Une société sans école, Paris 1971; P. Freire, La pedagogia degli oppressi, 1971.

${ }^{5}$ Cf. A.M. Mariani, Pedagogia e utopia. L'utopia pedagogica dell'educazione permanente, La Scuola, Brescia 1995.

${ }^{6}$ J. Delors (ed.), Learning: the Treasure within. Report to UNESCO of the International Commission on Education for the Twenty-frirst Century, Unesco 1966 (trad. it., Nell'educazione un tesoro, Armando Ed., Roma 1997).
} 
il tempo volge infatti alla società cosmopolita. Quella coesione civile, che l'apparato statale moderno era riuscito a garantire mediante la lunga e inconclusa fatica della democratizzazione, è insidiata nelle società post-moderne dal vistoso rimescolamento di culture, etnie, religioni nell'era della globalizzazione. Occorre dunque imparare a diventare cittadini del mondo, senza smettere di diventare al tempo stesso cittadini del proprio stato: l'utopia dei tempi nuovi, una scommessa seducente ma tutt'altro che scontata.

Il ruolo dell'educazione pubblica sposta il suo asse: pur tenendo fede al classico scopo di fornire strumenti per lo sviluppo personale e per la carriera professionale, il sistema educativo deve costruire e accrescere il senso di appartenenza a una comunità, la cui 'geometria' è sempre meno definita dalle tre classiche componenti: un territorio, una lingua, una religione, ed è sempre più caratterizzata invece dal melting pot etnico-culturale, dal mercato e dall'informazione transnazionali, dalla diversità etico-religiosa.

Viene così meno la plausibilità di modelli educativi collaudati in tempo di società monoculturali, e, dentro queste, la plausibilità del modello di trasmissione monoreligiosa o monoconfessionale ricalcato sullo stampo della scolarizzazione occidentale?

\section{Una tradizione originata nel passato, ma giocata sul futuro}

Un parallelo storico può giovare a situare la transizione presente, non però per proporre una analogia con cui simpatizzare oggi, ma per trarne semmai un argomento 'a contrario'. Usciva in Germania nel lontano 1799 un saggio dal titolo La cristianità ovvero l'Europa; autore ne era un giovane pensatore-poeta ventisettenne, un certo Georg Friederich von Hardenberg, più noto sotto lo pseudonimo di Novalis. Allarmato dalla devastazione etica-religiosa prodotta dal nuovo ordre de la raison diffuso dalla rivoluzione francese e, prima ancora, dagli 'effetti sciagurati' della riforma di Lutero, considerato il precursore dell'aborrito intellettualismo illuministico, il Novalis prospettava un ritorno al primato della

\footnotetext{
${ }^{7}$ Non entro qui nel merito dell'intenso inevitabile ripensamento di cui sono oggetto da anni i vari modelli di istruzione religiosa vigenti nei vari sistemi educativi del continente europeo. In altra sede ho espresso in proposito valutazioni e ipotesi, per es.: F.Pajer, Europa, scuola, religioni. Monoteismi e confessioni cristiane per una nuova cittadinanza europea, Sei, Torino 2005; Nuovi profili dell'istruzione religiosa in un'Europa pluralistica, in "Pedagogia e vita" 64 (2006) 2, p. 39-61; La scuola e le fedi, in "Educazione interculturale” (2009) 2, p. 211-226; Tendenze attuali e prospettive dell'istruzione religiosa scolastica nell'Unione europea, in “Orientamenti Pedagogici” (2010) 1, p. 27-50.
} 
religione: solo l'ordine della cristianità modellato su quello della felice simbiosi medievale, della res publica christiana, avrebbe potuto salvare l'Europa. Significativo l'incipit del suo saggio: "Erano belli, splendidi tempi quelli in cui l’Europa era una terra cristiana...”. Ma per Novalis non si trattava di un semplice ritorno romantico all'antico, bensì di un ribaltamento utopico, orientato alla creazione di una nuova cristianità, "che avrebbe dovuto ricostruire - scrive una Chiesa visibile senza riguardo a frontiere politiche, capace di accogliere nel suo grembo tutte le anime assetate dell'ultraterreno e di fare da mediatrice fra il mondo antico e il nuovo".

Sappiamo dalla storia come quell'ipotesi fascinosa non abbia avuto alcun seguito: poggiava su un presupposto non meno ideologico di quello che voleva combattere; riproponeva velleitariamente un ideale di cristianità in realtà mai esistito; anzi, la proposta prestava il fianco a strumentalizzazioni nostalgiche e reazionarie. e tuttavia il sogno di Novalis risulta emblematico in un tempo come il nostro, teso a rielaborare nuovi assetti mondiali dopo le cadute del muro di Berlino, dei blocchi ideologici, delle Twin Towers, proprio come l'Europa di duecento anni fa andava ricomponendo un 'nuovo ordine' dopo la rivoluzione francese e i miti prometeici dell'Aufklärung.

Quale lezione ci viene dunque per l'oggi? L'ha ricavata magistralmente il teologo Bruno Forte in una recente prolusione accademica parlando in tema di "radici ebraico-cristiane della cultura europea". Ne riprendiamo alcuni nodi. La 'casa europea' è stata la fucina di tutte le aspirazioni emancipatorie dell'età moderna, come anche di tanti totalitarismi ispirati da opposte ideologie sia ad Est che ad Ovest. Ideologie dichiarate cadute, ma che possono risorgere sotto altre spoglie, quelle religiose (o pseudo-religiose) comprese. Si capisce allora, dice Forte, "quale rischio comporterebbe il proporre per il futuro dell'Europa nuovi modelli ideologici, compreso quello di eventuali radici da ritrovare: l'eredità ebraico-cristiana potrà servire al superamento delle attuali difficoltà della coscienza europea solo se non sarà pensata in termini di ideologia rassicurante, di ritorno al passato".

Certo, permane innegabile che la Bibbia abbia giocato storicamente il ruolo di 'grande codice' della cultura europea (Frye Northrop), ma la vera posta in gioco è capire "se e in che misura la Bibbia possa ispirare oggi una prassi sociale e politica che soddisfi il bisogno diffuso di nuovo consenso etico". La risposta

${ }^{8}$ Cf. la prolusione tenuta in Assisi il 22 febbraio 2010, in occasione dell'inaugurazione del Centro studi sulle radici culturali ebraico-cristiane della civiltà europea dell’Università di Perugia. Uno stralcio del testo è stato ripreso dal Domenicale de Il Sole 24 ore, 21 febbraio 2010, p. 12; ma la sostanza della riflessione era già presente in un prcedente saggio dello stesso Forte: Cristianesimo ed Europa di fine millennio , in “Aggiornamenti sociali” 44 (1993) 7-8, p. 531-544. 
del teologo è senza esitazioni: se da una parte vanno riconosciute come dato storico le radici culturali ebraico-cristiane dell'Europa, non bisogna risuscitarle riproponendo oggi assetti politico-giuridico-educativi ormai superati. Superati non solo perché risultano anacronistiche e deleterie le alleanze strumentali tra stati e chiese, ma perché le stesse concezioni teologiche della fede biblico-cristiana, senza abdicare alla centralità della ragione, hanno abbandonato quelle pretese imperialistiche che facevano coincidere tout court fede e civiltà. Il potenziale delle radici ebraico-cristiane, afferma il teologo, "più che stare alle nostre spalle, ci provoca come qualcosa che sta davanti a noi e che ci chiede passi di libertà audace e scelte di intelligenza creativa".

Siamo dunque chiamati a un "ritorno al futuro", a un futuro non ancora disponibile ma reale, e non già a un passato estinto, riesumato ad arte solo nelle rivendicazioni nostalgiche dei nostrani 'teocon'. Un futuro che è possibile - e, per i credenti, doveroso - sperare, grazie al potenziale utopico insito nella rivelazione biblica. Che non è mai stata un sedativo per i perenni interrogativi dell'uomo, né un deterrente per le sue ambizioni più alte (vedi il "dominate la terra" di Genesi 1,28). Anzi, proprio l'annuncio delle "cose ultime” mobilita le energie per compiere appieno le "cose penultime"; la promessa dei beni ultimi non disincentiva affatto dall'impegno di costruire i beni penultimi, ma ne rinforza il ruolo propedeutico; non perché i beni penultimi sono 'laici' godono di minor dignità; nella logica dell'incarnazione la 'carne' della storia è luogo rivelatore del divino; presente dell'uomo e futuro di Dio sono certamente asimmetrici, ma l'uno è grembo generativo dell'altro. Nella logica del credere cristiano non è il cor inquietum che produce il domani utopico, ma è l'inaudito della resurrezione di Cristo che rende inquieto il cuore, caricandolo di una 'riserva escatologica' che lo libera fin da oggi dalle false sicurezze dell'ideologia e dell'idolatria, e lo stimola all'impegno per la liberazione del mondo. Il 'già' della storia è gravido del 'non ancora' escatologico.

Siamo distanti anni luce da quanto constatava - a carico del cristianesimo - lo storico olandese Johan Huizinga che, all'indomani della prima guerra mondiale, così riassumeva i percorsi utopici delle speranze umane9: "L’aspirazione a una vita più bella ha in ogni tempo visto dinanzi a sé tre vie. La prima conduceva fuori del mondo: era il sentiero della rinuncia. La vita più bella appare raggiungibile soltanto nell'aldi là; può essere soltanto una redenzione da ogni cosa terrena (...). Una strada battuta in ogni civiltà superiore, compreso il cristianesimo, che aveva inculcato questa tendenza come sostanza della vita individuale e sociale, così

${ }^{9}$ J. Huizinga, L'autunno del Medio Evo, Sansoni, Firenze 1953, p. $44-45$ (l'originale è del 1919). 
fortemente negli spiriti che per molto tempo fu preclusa la possibilità di seguire la seconda via. La seconda via è quella che conduce al miglioramento del mondo stesso. Il Medioevo l'ha conosciuta appena (...). Chi anelava al meglio e tuttavia non sapeva dire addio al mondo e alle sue gioie, non aveva altro davanti a sé che la disperazione. Quando si prenderà a battere la via del miglioramento positivo del mondo si inizierà una nuova epoca, in cui l'angoscia cederà il posto al coraggio e alla speranza. (...) Solo il secolo decimottavo erige a suo dogma centrale l'idea della perfettibilità dell'uomo e della società; il secolo seguente perderà soltanto quell'ingenuità, ma non il coraggio e l'ottimismo. Il terzo sentiero verso un modo più bello conduce nel regno dei sogni (...)".

Fin dai primi anni settanta si è rimessa a circolare in Europa e fuori una parola non nuova, ma assai inconsueta persino nel vocabolario quotidiano dei credenti: la parola speranza. Coniugata stancamente nei logori catechismi posttridentini delle cosiddette virtù teologali, quella parola aveva finito per perdere il suo significato teologico e persino la sua densità antropologica. Toccò al teologo evangelico Jürgen Moltmann rilanciarla nel vivo dei dibattiti alti dell'intellettualità europea e fu il reinizio fecondo di una "teologia politica"10, che andò presto a innervarsi nei solchi della teologia della liberazione latinoamericana. Nell'afflato ecumenico di queste teologie transconfessionali (e persino a-religiose, nel senso che incontravano Dio fuori dal tempio ma dentro la storia), il cristianesimo ridiventava per molti una utopia credibile, un progetto per cui valeva ancora la pena investire ideali, risorse e la vita stessa.

Ben a ragione una rivista internazionale di teologi, cristiani e non, può dichiarare oggi che "un altro mondo è possibile"11. Appena qualche decennio fa nell'immaginario collettivo dei credenti, uno slogan del genere, faceva pensare (ovviamente!) all'esistenza del mondo dell'aldilà, come ogni buon trattato di apologetica o di teologia fondamentale cercava di dimostrare. Oggi la percezione

${ }^{10}$ J. Moltmann, Teologia della speranza, Queriniana, Brescia 1971. Il teologo cristiano ha riconosciuto il suo debito al filosofo marxista Ernst Bloch che, nel 1959, pubblicava il suo noto Das Prinzip Hoffnung [trad. it. Il principio speranza, Garzanti, Milano 1994], opera alla quale si è ispirato, prendendone però le distanze dalla radicale e pervasiva immanenza intramondana. Lui stesso racconta il suo incontro col pensiero di Bloch: "Poi è entrato Bloch nel mio raggio visivo. Mi ricordo molto bene come io abbia passato un'intera vacanza nel Ticino in compagnia de Il principio speranza senza nulla vedere della bellezza delle montagne svizzere: Questa fu la mia prima impressione: Ma perché la fede cristiana si è lasciata sfuggire questo tema, che doveva essere il suo proprio tema?, dov'è andato a finire nel cristianesimo d'oggi lo spirito cristiano primitivo della speranza?” (cit. da R. Gibellini, La teologia del XX secolo, Queriniana, Brescia 1992, pp. 307-308).

${ }^{11}$ Cf. "Concilium”, rivista internazionale di teologia, anno XL, 5 (2004), monografia su Un altro mondo è possibile, a cura di L.C. Susin, J. Sobrino, F. Wilfred, pp. 172. 
della stessa parola ha cambiato contenuto semantico: non un altro mondo nell'aldilà, ma un altro mondo è possibile qui, su questo pianeta, in questa storia. Quanto dire che tutto ciò che è riconosciuto come creazione divina dipende anche dalle decisioni e dalle responsabilità umane.

I "possibili” della storia possono diventare realtà se c'è qualcuno che li sogna e opera di conseguneza per trasformarli in realtà. Sarà anche vero che le utopie non sono più di moda nel clima cinico che investe tutti. Ma è anche vero che una società senza utopie sarebbe una società senza speranza e quindi senza futuro. Proprio come recita un aforisma del poeta cantate Jim Morrison: "Ognuno di noi ha un paio d'ali, ma solo chi sogna impara a volare".

\section{Bibliography}

Augias C. e Mancuso V., Disputa su Dio e dintorni, Mondadori, Milano 2009. Beck U., La società cosmopolita. Prospettive dell'epoca postnazionale, Il Mulino, Bologna 2003.

Berger P.L., Questioni di fede. Una professione scettica del cristianesimo, Il Mulino, Bologna 2005.

Bloch E., Le principe Espérance, Gallimard, Paris 1976-1991.

Cullman O., Christ et le temps, Delachaux et Niestlé, Neuchatel 1959.

Duquoc Ch., Messianisme de Jésus et discrétion de Dieu, Labor et Fides, Genève 1984.

Forte B., Dove va il Cristianesimo? Queriniana, Brescia 2000.

Huizinga J., L'autunno del Medioevo, Sansoni, Firenze 1953.

Kaufmann F.X., Quale futuro per il Cristianesimo ? Queriniana, Brescia 2002.

Illich I., Une société sans école, Seuil, Paris 1971.

Mancuso V., L'anima e il suo destino, Raffaello Cortina, Milano 2007.

Mannheim K., Idéologie et Utopie, Marcel Rivière, Paris 1955.

Marazzi A., Lo sguardo antropologico. Processi educativi e multiculturalismo, Carocci, Roma 1999.

Mariani A.M., Pedagogia e utopia. L'utopia pedagogica dell'educazione permanente, La Scuola, Brescia 1995.

Moltmann J., Teologia della speranza, Queriniana, Brescia 1971.

Rorty R. e Vattimo G., Il futuro della religione, Garzanti, Milano 2005.

Schroeder R., Liquidazione della religione? Il fanatismo scientifico e le sue conseguenze, Queriniana, Brescia 2011. 